\title{
An Efficient Destructive Interference Based on Side Lobe Suppression Method in SONAR Beamforming
}

\author{
S. Vijayan Pillai, T. Santhanakrishnan* and R. Rajesh \\ Naval Physical and Oceanographic Laboratory, Kochi, Kerala, India
}

The manuscript was received on 18 June 2020 and was accepted after revision for publication as research paper on 16 April 2021.

\begin{abstract}
:
A novel beamforming technique that resembles the principle of interference is proposed for sonar arrays to suppress the side lobes while the main lobe is kept intact. It uses two window functions. The first one is a rectangular function that produces a primary beam pattern. A secondary new window function is derived and its beam pattern is steered such that the null or trough of the main lobe of the new window coincides with the peak or crest of the first side lobe of the rectangular window and so on to other major side lobes. Pattern multiplication was used to get a final beam pattern. The approach is simulated and verified through a sonar array with 24 hydrophone sensors.
\end{abstract}

\section{Keywords:}

sonar beamforming, side lobe suppression, product beamforming, pattern multiplication

\section{Introduction}

Surveillance against the surface and subsurface targets at sea and coast and the harbour are the most complex and challenging operational issues for the modern navy in the current turbulent global political climate. Most of the surveillance instruments fitted in seagoing vessels use different types of sonar systems. Sonar with a large number of hydrophone arrays is widely used to detect and process acoustic signals that arrive from various directions in the ocean due to their advantages having higher gain, directivity, and signal-to-noise ratio (SNR) compared to a single hydrophone [1]. Sonar beamforming is a technique for the estimation of the spatial Fourier wave number spectrum from measurements of a spatially varying acoustic field and thereby used to improve SNR for detecting targets and their bearing. Hypothetically, no sonar array can emit or receive all the energy in one preferred direction. Always some energy is inevitably distributed in other directions with relatively lower levels than the main

\footnotetext{
* Corresponding author: Naval Physical and Oceanographic Laboratory, Defence research and Development Organisation, Ministry of Defence, Government of India, Thrikkakara P.O., Kochi-682021, Kerala, India.E-mail: tsanthan@npol.drdo.in.ORCID 0000-0003-1583-9530.
} 
direction. These smaller crests are referred to as side lobes represented in decibel [dB] down from the main beam and represented as side lobe levels (SLL).

The critical design issue in beamforming is the selection of a proper window function, which depends on the geometry of the array. Some of the major problems existing in current sonar systems, which could not be addressed to date, are mainly due to hardware limitations. Distortion in the output of the beam pattern due to the amplitude, phase and position inaccuracies of individual hydrophones of the sonar array results in multiple display lines representing false targets in the sonar display, which is considered to be a major problem. Increased beamwidth and masking of weak targets by strong targets that are associated with window functions are other problems. The main goal of the beamformer is to generate a beam with a narrow main lobe and low side lobes, which unfortunately are two contrasting objectives.

Another vital problem in the beamforming technique is determining the shading coefficients or the weightage to be applied at the output of each hydrophone sensor with respect to the other hydrophones of the array for greater side lobe reduction [2]. These shading coefficients or weightage factors help to reduce the SLL at the expense of the main lobe widths [3] and array gain. The desired goal is to maximize the energy over the main beam and minimize the energy over the side lobes by holding the total energy to be conserved [4]. Practically, it is achieved by superimposing a suitable window of arbitrary shape over the main beam with a certain weighing factor along the spatial distribution of the array. Ideally, these shading coefficients should be robust enough to generate significant performance [5]. The array pattern should ideally yield a high array gain, directivity, low SLL, controllable beamwidth, good pattern characteristics and azimuthally better symmetry [6]. This is required to provide high bandwidth and quality sonar for operation in the ocean. It is apparent that the weight functions with finite aperture windows are the key deciding factors working to control and identify undesirable side lobes, perhaps with performance trade-offs.

Many types of weightage factors with window arrangements have been reported over the years to accomplish various optimizations. Finding or determining an optimum window function for some specific purposes has been the subject of research even today. Yang et al. [7] first reported an optimization method based on differential evolution to suppress the side lobes in linear antenna arrays. During the last decade, various works like utilization of differential evolution methods [8-10], genetic algorithms [11], simulated annealing [12, 13], particle swarm optimization [14-16], cuckoo search algorithm [17-18] and artificial neural networks [19-20] etc. were reported in the direction of reducing side lobes for the desired pattern at the interested operating frequency band. However, almost all of them were learnt to have performed well with the applications in their desired operational scenario. It is understood that the different amplitude windows yield far-field beam patterns with different side lobe levels and beamwidths. It is also seen that, as side lobe levels decrease, beamwidth generally increases. Most of these techniques were verified and implemented in RF antenna research and developments [7]. But only a few of them were successfully tested in sonar beamforming.

The most successful and used method in sonar beamforming is based on the Dolph-Chebyshev window. The main advantage of this method is its capability to optimize such far-field beam patterns which enable to achieve the narrowest main lobe beamwidth for any specified side lobe level and also for the lowest possible side lobe level relative to the main lobe. On the other hand, the disadvantage of this method is that as the weighting factors of each element in the array are not equal, the effective 
sensitivity gets degraded [21-26]. Further, the window function gets influenced largely by the hydrophone elements in the centre of the array. Perhaps this method is good for beamforming for spatial imaging applications, but not so good for cases where control of frequency artefacts is essential, mainly when they are operated for sonar beamforming. This is because in practical sonars having a large sensor array, various inaccuracies, like position error in hydrophone elements spacing, errors in amplitude and phase, are normally encountered. This is in addition to the clutter environments with high ocean dynamics where the sonar systems are operated, making it inadequate, hence there is a need for more attempts to reduce the sparse in window functions, especially for sonar beamforming so that a broader scope can be accomplished. Another important method utilized for reducing the SLL is based on product theorem [27], wherein the elements in the array are considered to be directional to achieve the final beam, which is practically very complex.

This paper introduces a relatively simple and computationally efficient method in which robust rectangular weighing vectors were used to obtain the fundamental beam pattern. Thereafter, another new window function is used in such a way that two sets of window functions were operated for each aperture window innovatively for the final beamforming. The crests of two windows are multiplied to obtain the primary main beam and its veracity is maintained based on the constructive interference principle used in optics. Likewise, the crest on one beam pattern with the trough of the second window is multiplied to obtain the side lobes based on the destructive interference principle. Thereby, it greatly simplifies the difficulties that arise randomly in most of the sonar beamforming techniques. Numerical examples for a sonar array with 24 hydrophone elements are presented exhibiting ultra-low side lobes. The results obtained with the present method are compared with those obtained by other beamforming techniques to highlight the improvements and the robustness of the present method. Section 2 of the paper examines the properties of rectangular and DolphChebyshev window functions to demonstrate how familiar functions succeed or fail to meet the objectives of obtaining lower SLL, narrow main beam, etc. The proposed window function is described in Section 3 along with the beamformer. Simulation and implementation are described in Section 4 followed by the conclusion in Section 5 .

\section{Methodology}

The basic sonar system consists of the following important functional blocks as illustrated in Fig. 1.

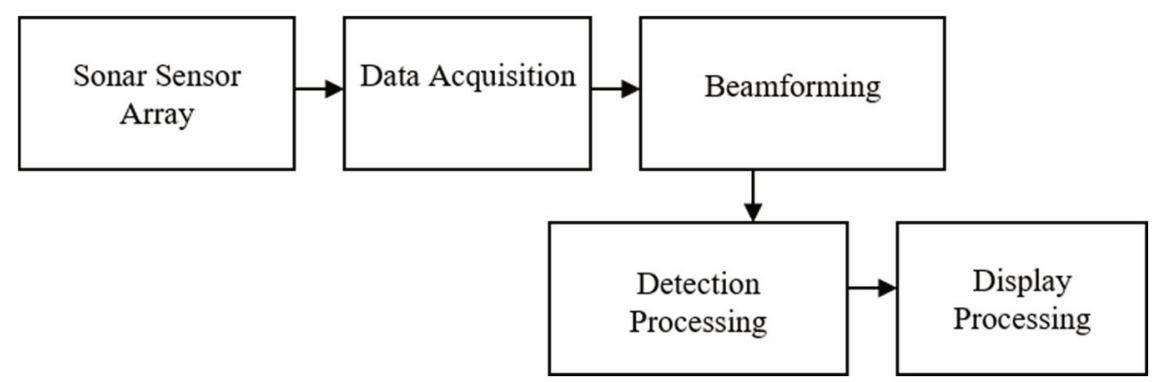

Fig. 1 Basic building blocks of a sonar array

The sonar sensor array consists of a set of hydrophone elements arranged optimally. The data acquisition block receives the signals from individual hydrophones 
and carries out signal condition in both time and spectral domain. The most important block is the beamformer which provides the direction of arrival and improves the SNR of an incoming acoustic signal. The detection processor block provides the detection with control on the given probability of detection and probability of false alarm. The display processor block quantizes the detector output into a presentable form for display. The present work mainly focuses on the beamformer concepts and their effects.

Consider a linear array of hydrophones with an inter-element spacing of $\lambda / 2$, where $\lambda$ is the wavelength that corresponds to the maximum acoustic frequency of the considered acoustic band in water, which is received from a far-field acoustic source as shown in Fig. 2. Consider an acoustic source is placed at the broad-side of a sonar array and its wave impinges perpendicular to the plane of the sonar array, then the outputs of each hydrophone are in phase and will add up coherently. Correspondingly, the beam pattern will be formed with the beam centring at $0^{\circ}$, as shown by the solid line in the bottom curve of Fig. 2. As the acoustic source is moved around the sonar array or when the sonar array is rotated with respect to the acoustic source and when the acoustic wave impinges at an angle with respect to the normal of the hydrophone elements, then the hydrophones receive signals with different time delays. In such a situation, the outputs of each hydrophone no longer add coherently and the peak gets shifted after the adder. To cater to this, suitable mathematical manipulations or beamforming techniques need to be applied to ensure a better beam pattern with higher gain from all the hydrophone elements.

In this context, the two most familiar window functions that are frequently used by beamforming designers are examined here to provide a background for discussions. Window functions are used in harmonic analysis to reduce the undesirable effects related to spectral leakage. Window functions influence many attributes that include detectability, resolution, dynamic range and ease of implementation. The SLL is usually expressed as a ratio of the power density in that lobe relative to that of the main lobe expressed in $\mathrm{dB}$. On the other hand, the SLL is the amplitude of the side lobe normalized to the peak of the main beam. The highest side lobe is usually the lobe closest to the main beam. The SLL can be controlled by employing proper weightage to individual hydrophone elements. There are many popular window functions namely Rectangle, Triangle, $\cos ^{\alpha}(t)$, Hann, Hamming, short cosine series, Blackman, HarrisNutall, Sampled Kaiser-Bessel, parabolic, Riemann, Tukey, Bohman, Poisson, HannPoisson, Cauchy, Gaussian, Dolph-Chebyshev, Kaiser-Bessel Barcilon-Themes etc., which are mostly used for sonar beamforming [26].

The main deciding factors that highlight the efficiency of a particular window function for any application are the highest side lobe level [dB], side lobe fall rate [dB/Octave], coherent gain, $3.0 \mathrm{~dB}$ beamwidth, etc. The theoretical values of these factors for some of the window functions are given in Tab. 1. The lowest value for highest SLL, highest fall rate in the side lobes, maximum coherent gain and minimum beamwidth are the most desirable outputs from any of the beamformer.

\subsection{Rectangular Window}

The rectangle window is unity over the observation interval and can be thought of as a gating sequence applied to the data so that they are of finite extent. For a simple linear array of $N$ hydrophone elements as shown in Fig. 2, the window is defined as

$$
w(n)=1 \quad \text { for } n=-\frac{N}{2}, \cdots,-1,0,+1, \cdots,+\frac{N}{2}
$$




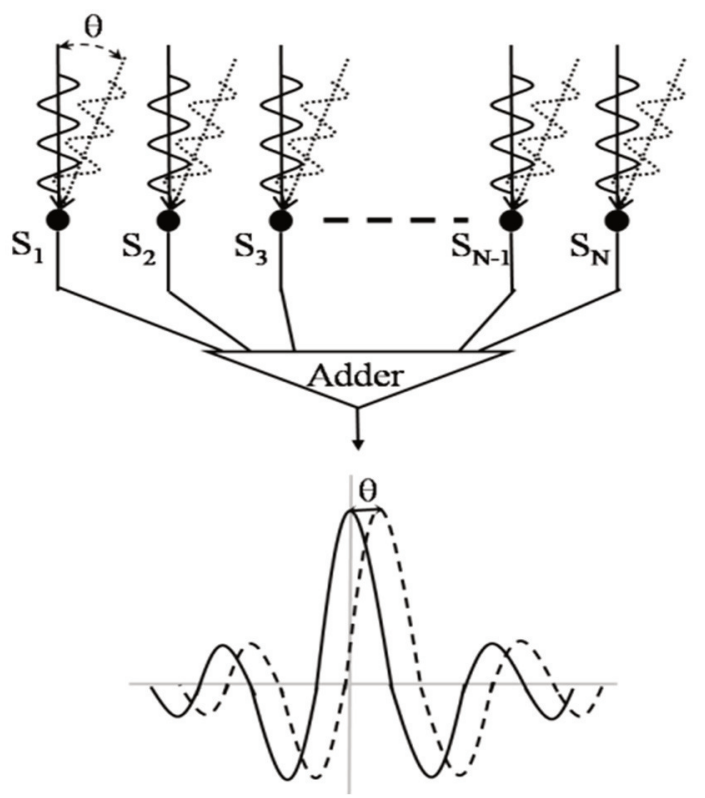

Fig. 2 Schematic of time-domain beamforming with broadside and off-broadside targets

At the output of the adder, the output $B(\theta)$ will take the simple arithmetic sum of the outputs of the individual hydrophone elements, scaled by a factor $1 / N$ so that the polar response maximum is unity, irrespective of the number of hydrophone elements in the array [25],

$$
B(\theta)=\frac{1}{N} \cdot \frac{\sin \left(\frac{N \pi d}{\lambda} \sin \theta\right)}{\sin \left(\frac{N d}{\lambda} \sin \theta\right)}
$$

For the inter-element spacing distance between the hydrophone elements, $d=\lambda / 2$,

$$
B(\theta)=\frac{1}{N} \cdot \frac{\sin \left(\frac{N \pi}{2} \sin \theta\right)}{\sin \left(\frac{\pi}{2} \sin \theta\right)}
$$

The beam pattern obtained using the rectangular window function for a sonar array with 24 hydrophone elements separated by an inter-element spacing of $\lambda / 2$ is shown in Fig. 3. All the hydrophone elements in the array are given an equal weightage. The obtained beam pattern exhibits the first SLL of $-13 \mathrm{~dB}$ below the peak of the main lobe and the second side lobe at $-17.68 \mathrm{~dB}$ below. The width of the main beam is $\sim 4.2^{\circ}$. The first nulls are seen at $\sim \pm 4.8^{\circ}$, second nulls are seen at $\sim \pm 9.6^{\circ}$ and the third nulls are seen at $\sim \pm 14.5^{\circ}$. Since all the hydrophone elements with equal weights have contributed to beamforming, the array sensitivity is observed to be maximum with a high gain in array SNR of $13.8 \mathrm{~dB}$. As seen from Fig. 3, the higher SLL is the most undesirable feature of this window function. 


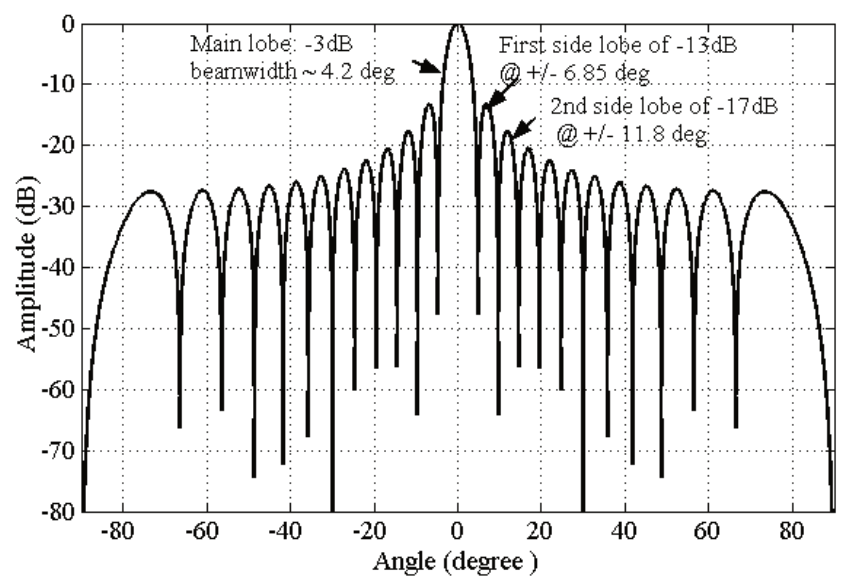

Fig. 3 Beam pattern for rectangular window function with 24 elements array

\subsection{Dolph-Chebyshev Window}

The criterion addressed by the Dolph-Chebyshev window is that its Fourier transform exhibits the narrowest main lobe and adjustable SLLs. The Fourier Transform of the window is a mapping of the $N$-th order algebraic Chebyshev polynomial to the $N$-th order trigonometric Chebyshev polynomial by a relationship $T N(x)=\cos (N q)$. The Dolph-Chebyshev window is defined in terms of uniformly spaced samples of its Fourier Transform as

$$
W(k)=\frac{\cosh \left[N \cosh ^{-1}\left(\beta \cos \frac{\pi k}{N}\right)\right]}{\cosh \left(N \cosh ^{-1} \beta\right)} \text { for } x>1
$$

where $\cosh ^{-1} x=\ln \left(x+\sqrt{x^{2}-1}\right)$

$$
W(k)=\frac{\cos \left[N \cos ^{-1}\left(\beta \cos \frac{\pi k}{N}\right)\right]}{\cosh \left(N \cosh ^{-1} \beta\right)} \text { for } x \leq 1
$$

where $\cos ^{-1} x=\frac{\pi}{2}-\tan ^{-1} \frac{x}{\sqrt{1-x^{2}}}$.

The term $\beta$ is defined as $\beta=\cosh \left[1 / N \cosh ^{-1} 10^{\alpha}\right]$, where $\alpha=-A / 16.6$. Here, $A$ is SLL represented in $\mathrm{dB}$. The discrete-time Dolph-Chebyshev window is obtained by taking the inverse discrete Fourier transform (DFT) of $W(k)$ and normalizing the result to a peak value of 1 as

$$
w(n)=\sum_{k=0}^{N-1} W(k) \exp \left(j \frac{2 \pi}{N} n k\right) \text { for } n=0, \cdots, N-1
$$

For a sonar array with 24 hydrophone elements, the obtained discrete-time Dolph-Chebyshev window is shown in Fig. 4 and indicates the weighing factor to each hydrophone elements in the array. 


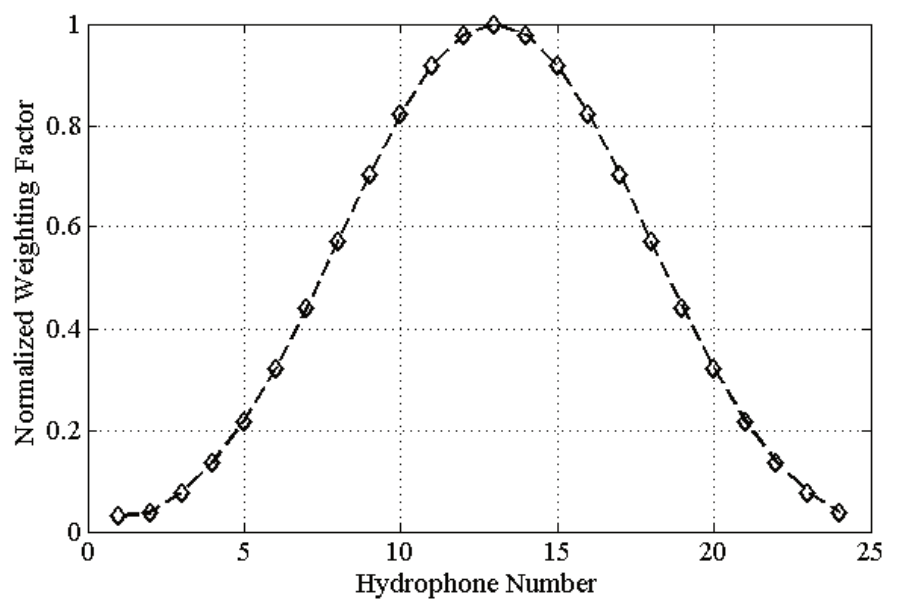

Fig. 4 The Dolph-Chebyshev window used for a sonar array with 24 elements

The beam pattern obtained using Dolph-Chebyshev window function for a sonar array with 24 hydrophones separated by an inter-element spacing of $\lambda / 2$ is shown in Fig. 5.

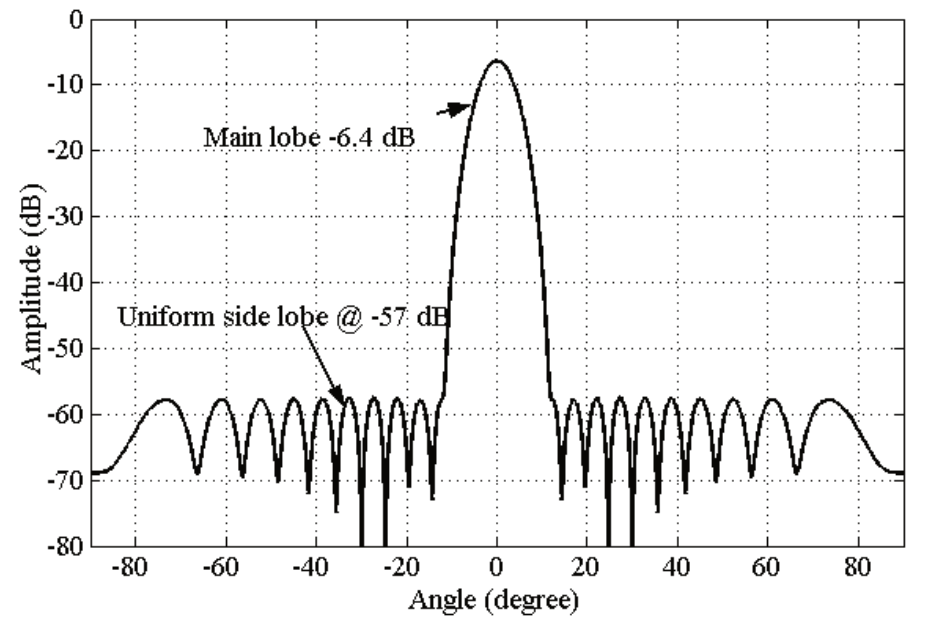

Fig. 5 Beam pattern for Dolph-Chebyshev window with 24 elements array

As seen from Fig. 5, this window function exhibits equal ripples at the specified SLL. The constant level side lobes levels are inherited from the Chebyshev polynomial and as such contain impulses in its time series. These impulses are located at the window boundaries. Windows with constant SLL, while optimal in the sense of equal ripple approximation, are suboptimal in terms of their integrated SLL. It is observed in Fig. 5 that a uniform SLL is formed approximately at $-57 \mathrm{~dB}$ for a given $\alpha=3.5$. The $3 \mathrm{~dB}$ beamwidth increased with a factor of approximately 1.7 as that of the rectangular window. However, a reduction in array gain by approximately a factor of 0.45 is observed, which is undesirable. 


\section{Present Method}

In the cases of the rectangular window or Dolph-Chebyshev window methods, it is assumed that the individual hydrophone elements have an omnidirectional response. The parameters of the beam pattern can be improved using directional hydrophone elements and product theorem [27]. However, the construction of identical directional hydrophones in a large number especially on the lower frequency side of an acoustic spectrum for a large sonar array is complex and costly. The advantage of the product theorem is adopted in the present method but using only the omnidirectional hydrophones. On the other hand, two different window functions are used independently on the entire sonar array and thereby two different beam patterns are generated. A final beam pattern is obtained by the cross product of these two beam patterns innovatively as explained in the following lines. The new beamformer scheme is shown in Fig. 6.

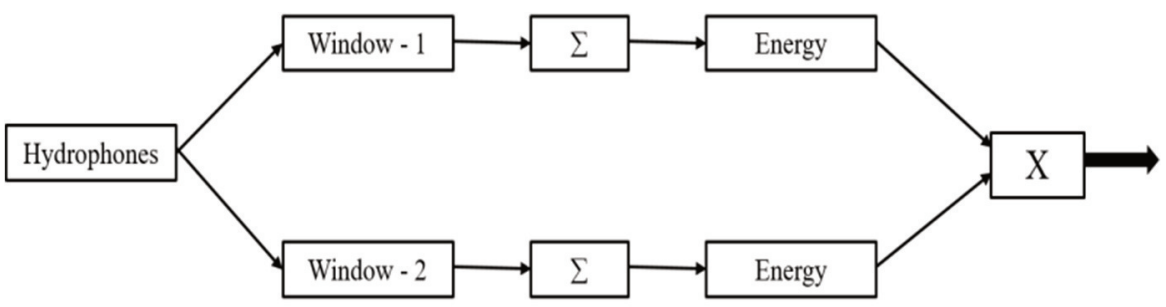

Fig. 6 Schematic of the present beamforming method

It is learnt from Section 2.1 and previous discussions that beam pattern obtained using a rectangular window has minimum beamwidth, maximum array sensitivity and maximum tolerance to array parameters while having the only disadvantage of higher SLL. Therefore, the rectangular window is chosen to be one of the window functions to be used in the present method. The second window function is derived from the Dolph-Chebyshev window with $\alpha=1.414$. This second window is selected to form a beam pattern in such a way that the trough positions belonging to the side lobes of this beam pattern coincide with the crest positions belonging to the side lobes of the rectangular window without affecting the main lobe with the use of null steering. The corresponding discrete-time Dolph-Chebyshev window obtained by taking the shifted inverse DFT of $W(k)$ and normalizing the result to a peak value of 1 are

$$
\begin{gathered}
w(n)=\sum_{k=0}^{N-1} W(k) \exp \left[j \frac{2 \pi}{N}(n+1) k\right] \text { for } n=0, \cdots, N / 2-1 \\
w(n)=\sum_{k=0}^{N-1} W(k) \exp \left(j \frac{2 \pi}{N} n k\right) \text { for } n=N / 2, \cdots, N-1
\end{gathered}
$$

The discrete-time Dolph-Chebyshev window obtained for the considered sonar array with 24 hydrophone elements is shown in Fig. 7 and provides the weighing factor to all the hydrophone elements in the array.

\section{Results and Discussion}

In the present method, pattern multiplication is employed to get the best advantage of the rectangular window. Fig. 8 shows the obtained beam patterns with the rectangular and the new windows. It is seen from Fig. 8 that the beam pattern has been steered in 
such a way that the null or trough of the main lobe belonging to the new window coincides with the peak or crest of the first side lobe belonging to the rectangular window. Similar null coinciding points are seen for a few other major side lobes.

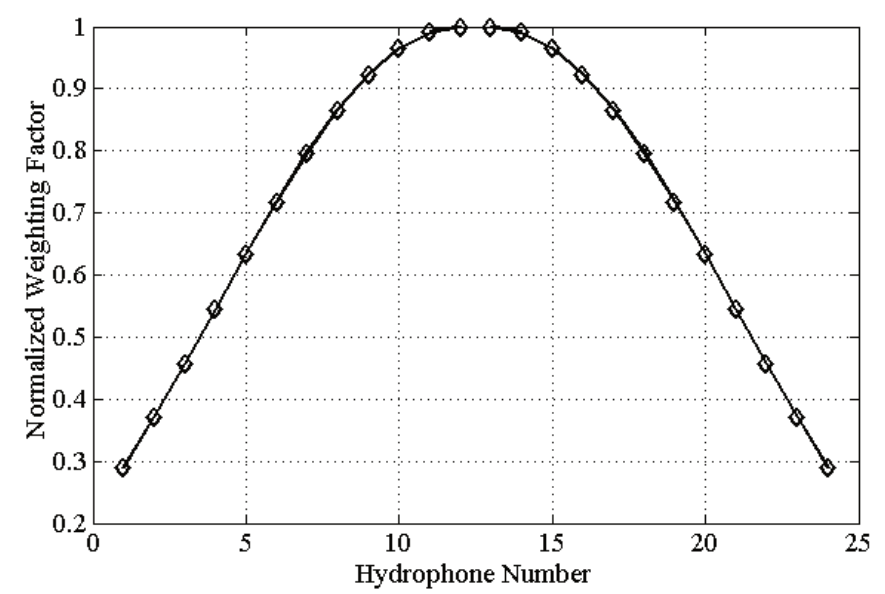

Fig. 7 New window used for a sonar array with 24 hydrophone elements

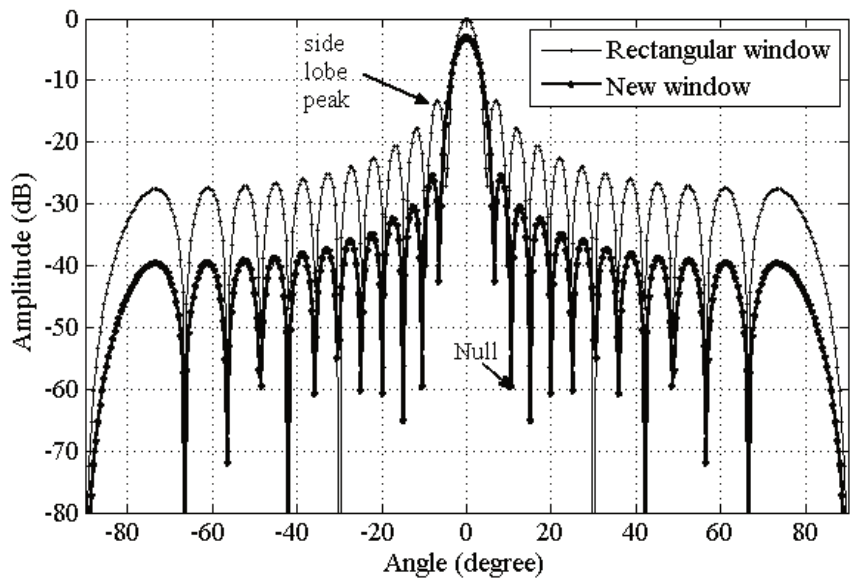

Fig. 8 Beam patterns of rectangular and new windows

Fig. 9 illustrates the final product beam pattern obtained using the present approach by pattern multiplication of beam patterns corresponding to the rectangular and new window-based beams shown in Fig. 8. It is noticeable that the steered nulls of the new window brought down the prominent side lobes of the rectangular window without affecting the main beamwidth and array sensitivity. On the other hand, Fig. 9 demonstrates the efficacy of the present method in suppressing the side lobes in beamforming.

An ideal beamformer expects a planar wavefront. On the other hand, all the hydrophone elements in the array are expected to face the wavefronts in the same phase for forming a perfect broadside beam. For a given wavelength $\lambda$ and aperture length $D$, the planar wavefront can be achieved when the array is at a far-field, i.e. a distance greater than $2 D^{2} / \lambda$. A wavefront with wavelength $\lambda$ travelling through a distance of $d$ 
will experience a phase change of $2 \pi d / \lambda$. On the other hand, a wave with a wavelength of $0.5 \mathrm{~m}$ can experience a phase change of $\pm 0.0628 \mathrm{rad}\left( \pm 3.6^{\circ}\right)$ which corresponds to an error of $\pm 0.5 \mathrm{~mm}$ in its sensor element position. For the present sonar array with 24 hydrophone elements and $\lambda / 2$ inter-element spacing, the far-field distance is $288 \lambda$. The main factor which affects the beam pattern is the phase error manifesting out of variation in inter-element distance referred here as 'the location' and planarity of array aperture. Yet, the other array parameter which affects the beam pattern is the variation between the sensitivity of the hydrophone elements. An ideal beam is formed in broadside only when the hydrophone element sensitivity is omnidirectional and identical.

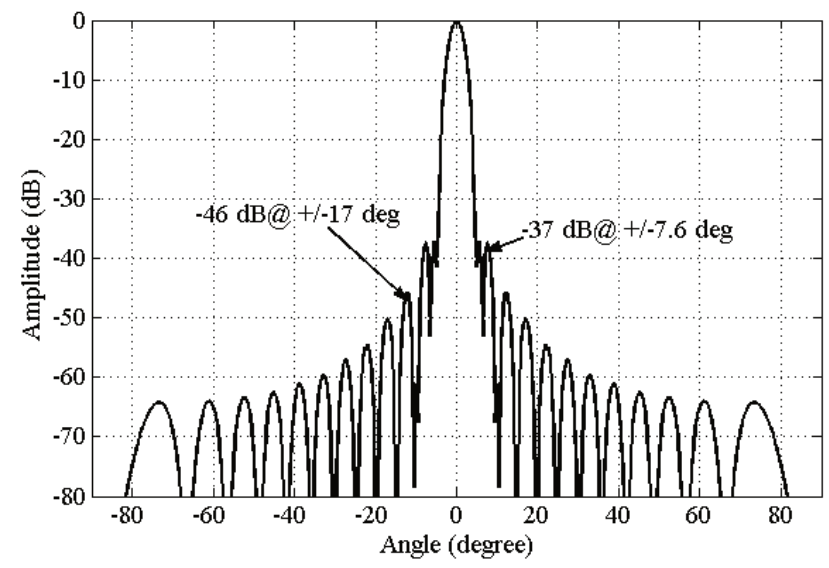

Fig. 9 The resultant beam pattern obtained using the present method

To see the effects of the above-mentioned uncertainties in the beamforming, a sonar array with 24 hydrophone elements and a cumulative amplitude error of $10 \%$ due to some of the inevitable uncertainties like the error in the amplitude of individual hydrophones due to the inaccuracies in $\lambda / 2$ location, array integrity and sensitivity mismatch etc. is considered for analysis. The magnitude of perturbation observed in the case of the rectangular window and Dolph-Chebyshev window-based beamformer is shown in Figs 10 and 11, respectively.

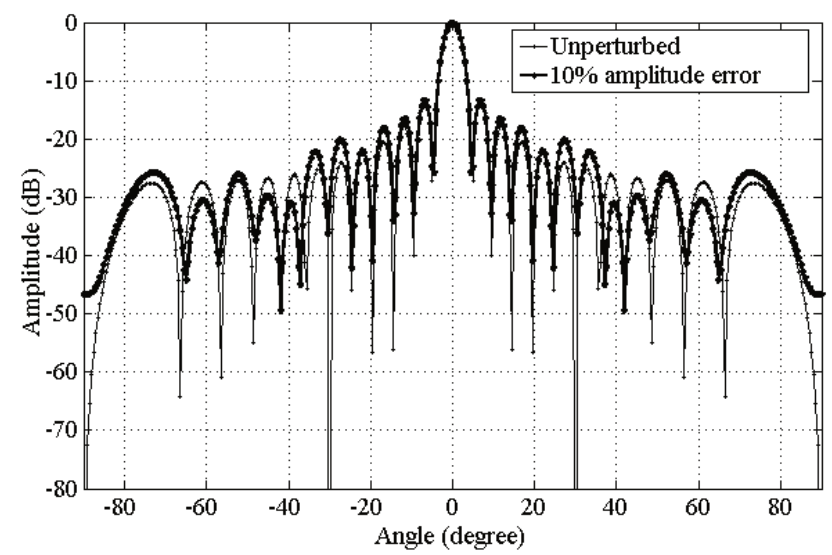

Fig. 10 Magnitude of perturbation in beam patterns with rectangular window 


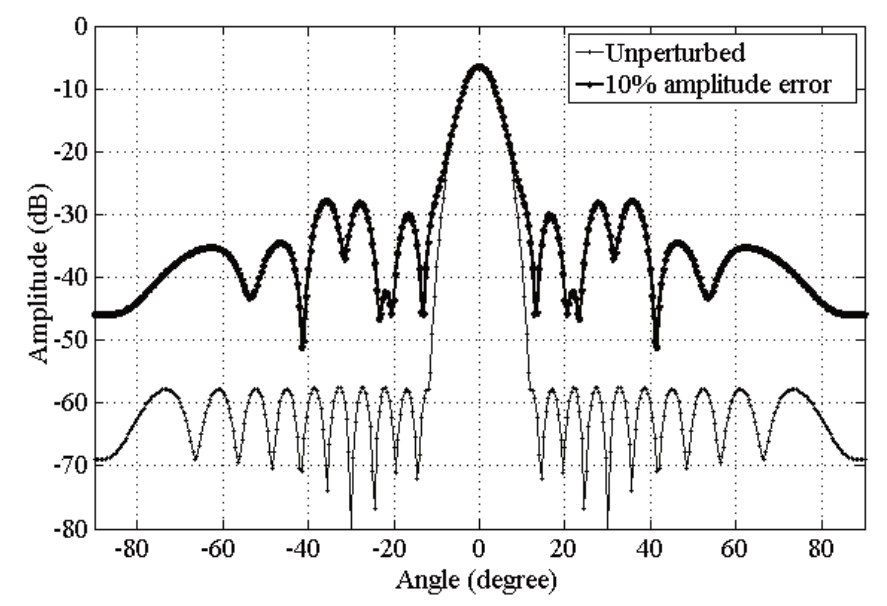

Fig. 11 Magnitude of perturbation in beam patterns with Dolph-Chebyshev window

The graphs shown in Figs 10 and 11 obtained by simulation show that the magnitude of perturbation in rectangular window-based beamformer is much lower than that of the Dolph-Chebyshev window-based beamformer. In other words, the rectangular window-based beamformer is more robust than the Dolph-Chebyshev window-based beamformer. However, it is obvious from Figs 10 and 11 that the side lobes in both the cases increase even for a small $10 \%$ perturbation in amplitude, making both beamformers unsuitable in most of the sonar systems as small inaccuracies in locations of individual hydrophones, identical sensitivity and other inaccuracies introduced during the manufacturing of hydrophones and their integration are unavoidable.

The simulation result of the beamformer with the new method, for the same array and perturbations, is shown in Fig. 12. It is observed that there is no considerable distortion in lobes up to $\pm 30^{\circ}$. This is the main advantage of destructive interferencebased pattern multiplication adopted in the present method. However, beyond $\pm 30^{\circ}$, marginal increases in the side lobes are noticed, which does not affect any sonar operation as they are far away from the main look angle.

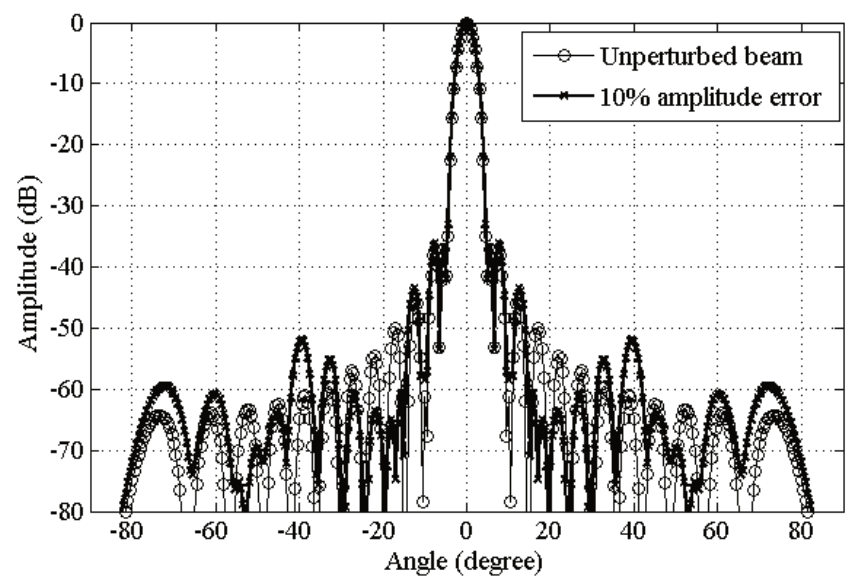

Fig. 12 Magnitude of perturbation in beam patterns in the new method 
Performance comparison of different beamforming methods is carried out and the results are tabulated in Tab. 1, which highlights the highest SLL, SLL fall rate, effective utilization factor of the array elements and increase in $3 \mathrm{~dB}$ beamwidth of the main lobe relative to that of the rectangular window.

Tab. 1 Comparison of beamforming performance parameters with different window functions [26]

\begin{tabular}{|l|c|c|c|c|}
\hline Window & $\begin{array}{c}\text { Highest } \\
\text { SLL [dB] }\end{array}$ & $\begin{array}{c}\text { Side Lobe } \\
\text { Fall rate } \\
\text { [dB/Octave] }\end{array}$ & $\begin{array}{c}\text { Effective } \\
\text { utilization } \\
\text { factor }\end{array}$ & $\begin{array}{c}\text { Increase in } \\
\text { 3 dB Beamwidth }\end{array}$ \\
\hline Rectangle $\times$ Rectangle & -26 & -12 & 1.000 & 1.000 \\
\hline $\begin{array}{l}\text { Present Method } \\
\text { Rectangle } \times \text { New Win- } \\
\text { dow })\end{array}$ & -38 & -14 & 0.956 & 1.087 \\
\hline
\end{tabular}

\section{Conclusions}

A simple concept of beamforming in the sonar system is reported in this paper. The method uses pattern multiplication of two different beam patterns which are obtained using a rectangular and a modified null steered Dolph-Chebyshev window functions. The pattern multiplication is implemented in such a way that it follows the destructive interference principle to reduce the SLLs. The advantages of the new method are demonstrated in comparison to other popular methods used in beamforming. The stability of the present technique is verified with some of the inevitable uncertainties, such as the error occurrence in the amplitude of individual hydrophones due to the inaccuracies in $\lambda / 2$ location, array integrity and sensitivity mismatch, etc. This new technique is found to be robust in sonar beamforming which can tackle problems associated with array sensitivity, intolerance to array parameters and can yield better performance. A close to unity value in effective utilization factor and beamwidth was achieved. The deterioration in other parameters has not been frequently observed. However, the deterioration effects cannot be ignored if the optimization tries to align all the nulls of one window function to the peaks of the other. The authors are carrying out a sustained investigation in this aspect and the result would be communicated in succession.

\section{Acknowledgements}

The authors thank Dr. Sameer V. Kamath, Director General of Naval Systems and Materials, DRDO, Ministry of Defence for the support and permission to publish the paper in Advances in Military Technology journal.

\section{References}

[1] ZIOMEK, L.J. An Introduction to Sonar Systems Engineering. New York: CRC Press, 2017. ISBN 978-1-49-877872-5.

[2] BUTLER, J.L. and C.H. SHERMAN. Transducers and Arrays for Underwater Sound (Modern Acoustics and Signal Processing). $2^{\text {nd }}$ ed. London: Springer, 2016. ISBN 978-3-31-939044-4. 
[3] LURTON, X. An Introduction to Underwater Acoustics: Principles and Applications. $2^{\text {nd }}$ ed. London: Springer, 2010. ISBN 978-3-54-078480-7.

[4] BALANIS, C.A. Antenna Theory: Analysis and Design. $4^{\text {th }}$ ed. Hoboken: Wiley, 2005. ISBN 978-1-11-864206-1.

[5] VESA, A. and A. IOZSA. Direction-of-Arrival Estimation for Uniform Sensor Arrays. In: Proceedings of the $9^{\text {th }}$ International Symposium on Electronics and Telecommunications. Timisoara: IEEE, 2010, pp. 249-252. DOI 10.1109/ISETC.2010.5679326.

[6] WAITE, A.D. Sonar for Practising Engineers. $3^{\text {rd }}$ ed. New York: Wiley, 2002. ISBN 978-0-47-149750-9.

[7] YANG, S., Y.B. GAN and A. QING. Sideband Suppression in Time-Modulated Linear Arrays by the Differential Evolution Algorithm. IEEE Antennas and Wireless Propagation Letters, 2002, 1(1), pp. 173-175. DOI 10.1109/lawp.2002.807789.

[8] AKSOY, E. and E. AFACAN. Thinned Non-Uniform Amplitude TimeModulated Linear Arrays. IEEE Antennas and Wireless Propagation Letters, 2010, 9, pp. 514-517. DOI 10.1109/lawp.2010.2051312.

[9] ZHU, Q., S. YANG, L. ZHENG and Z. NIE. Design of a Low Side Lobe Time Modulated Linear Array with Uniform Amplitude and Sub-Sectional Optimized Time Steps. IEEE Transactions on Antennas Propagation, 2012, 60(9), pp. 44364439. DOI 10.1109/tap.2012.2207082.

[10] MANDAL, S.K., G.K. MAHANTI and R. GHATAK. Differential Evolution Algorithm for Optimizing the Conflicting Parameters in Time-Modulated Linear Array Antennas. Progress in Electromagnetics Research B, 2013, 51, pp. 101118. DOI 10.2528/pierb13022710.

[11] GOSWAMI, B. and D. MANDAL. A Genetic Algorithm for the Level Control of Nulls and Side Lobes in Linear Antenna Arrays. Journal of King Saud University - Computer and Information Sciences, 2013, 25(2), pp. 117-126. DOI 10.1016/j.jksuci.2012.06.001.

[12] FREETHY, S.J., V.F. SHEVCHENKO and R.G.L. VANN. Optimization of Wide Field Interferometric Arrays via Simulated Annealing of a Beam Efficiency Function. IEEE Transactions on Antennas and Propagation, 2012, 60(11), pp. 5442-5446. DOI 10.1109/tap.2012.2207936.

[13] ZANGENE, A., H.R. DALILI OSKOUEI and M. NOURHOSEINI. Reduction of Side Lobe Level in Non-Uniform Circular Antenna Arrays Using the Simulated Annealing Algorithm. Journal of Electrical and Electronics Engineering Research, 2014, 6(2), pp. 6-12. DOI 10.5897/jeeer2014.0504.

[14] ROBINSON, J. and Y. RAHMAT-SAMII. Particle Swarm Optimization in Electromagnetics. IEEE Transactions on Antennas and Propagation, 2004, 52(2), pp. 397-407. DOI 10.1109/tap.2004.823969.

[15] KHODIER, M.M. and C.G. CHRISTODOULOU. Linear Array Geometry Synthesis with Minimum Side Lobe Level and Null Control Using Particle Swarm Optimization. IEEE Transactions on Antennas and Propagation, 2005, 53(8), pp. 2674-2679. DOI 10.1109/tap.2005.851762.

[16] LIANG, L., J. SUN, H. LI, J. LIU, Y. JIANG and J. ZHOU. Research on Side Lobe Suppression of Time-Modulated Sparse Linear Array Based on Particle 
Swarm Optimization. International Journal of Antennas and Propagation, 2019, 2019, 7130106. DOI 10.1155/2019/7130106.

[17] YANG, X.S. and S. DEB. Multi-objective Cuckoo Search for Design Optimization. Computers \& Operations Research, 2013, 40(6), pp. 1616-1624. DOI 10.1016/j.cor.2011.09.026.

[18] ONG, P. Adaptive Cuckoo Search Algorithm for Unconstrained Optimization. The Scientific World Journal, 2014, 2014, 943403. DOI 10.1155/2014/943403.

[19] GHAYOULA, R., N. FADLALLAH, A. GHARSALLAH and M. RAMMAL. Design, Modelling, and Synthesis of Radiation Pattern of Intelligent Antenna by Artificial Neural Networks. ACES Journal, 2008, 23(4), pp. 336-344. ISSN 1054-4887.

[20] ZAHARIS, Z.D., K.A. GOTSIS and J.N. SAHALOS. Adaptive Beamforming with Low Side Lobe Level Using Neural Networks Trained by Mutated Boolean PSO. Progress in Electromagnetics Research, 2012, 127, pp. 139-154. DOI 10.2528/pier12022806.

[21] DOLPH, C.L. A Current Distribution for Broadside Arrays Which Optimizes the Relationship between Beam, Width and Side-Lobe Level. Proceedings of the IRE, 1946, 34(6), pp. 335-348. DOI 10.1109/jrproc.1946.225956.

[22] NUTTALL, A.H. Generation of Dolph-Chebyshev Weights Via a Fast Fourier Transform. Proceedings of the IEEE, 1974, 62(10), pp. 1396-1396. DOI 10.1109/proc.1974.9634.

[23] ZIELINSKI, A. An Efficient Method of Dolph-Chebyshev Beamforming. In: H.M. MERKLINGER, ed. Progress in Underwater Acoustics. Boston: Springer, 1987, pp. 759-764. ISBN 978-1-46-129043-8.

[24] MOHAN, K.N., D. KANNADASSAN and S.R. ZINKA. Design and Implementation of Dolph Chebyshev and Zolotarev Circular Antenna Array. Indian Journal of Science and Technology, 2016, 9(36), pp. 1-9. DOI 10.17485/ijst/2016/v9i36/102137.

[25] BLONDEL, P. The Handbook of Sidescan Sonar. New York: Springer, 2009. ISBN 978-3-54-042641-7.

[26] POULARIKAS, A.D. The Handbook of Formulas and Tables for Signal Processing. Heidelberg: Springer, 1999. ISBN 978-3-54-064834-5.

[27] URICK, R.J. Principles of Underwater Sound. $3^{\text {rd }}$ ed. New York: McGraw-Hill, 1983. ISBN 978-0-07-066087-8. 